\title{
Asymptotic Safety, Asymptotic Darkness, and the hoop conjecture in the extreme UV
}

\author{
Sayandeb Basu \\ Physics Department, University of the Pacific 3601 Pacific Avenue, Stockton, CA 95211 \\ and \\ David Mattingly \\ Physics Department, University of New Hampshire, Durham, NH 03824
}

\begin{abstract}
Assuming the hoop conjecture in classical general relativity and quantum mechanics, any observer who attempts to perform an experiment in an arbitrarily small region will be stymied by the formation of a black hole within the spatial domain of the experiment. This behavior is often invoked in arguments for a fundamental minimum length. Extending a proof of the hoop conjecture for spherical symmetry to include higher curvature terms we investigate this minimum length argument when the gravitational couplings run with energy in the manner predicted by asymptotically safe gravity. We show that argument for the mandatory formation of a black hole within the domain of an experiment fails. Neither is there a proof that a black hole doesn't form. Instead, whether or not an observer can perform measurements in arbitrarily small regions depends on the specific numerical values of the couplings near the UV fixed point. We further argue that when an experiment is localized on a scale much smaller than the Planck length, at least one enshrouding horizon must form outside the domain of the experiment. This implies that while an observer may still be able to perform local experiments, communicating any information out to infinity is prevented by a large horizon surrounding it, and thus compatibility with general relativity can still be restored in the infrared limit.
\end{abstract}

\section{INTRODUCTION}

The conjecture that the spectrum of particle states near the Planck scale is dominated by black holes, so called "asymptotic darkness", is an old one. Proof of such a conjecture requires a particular theory of quantum gravity, however one can make an argument with solely general relativity and quantum mechanics. The argument relies on Thorne's hoop conjecture [1]: If an amount of energy or matter $E$ is compressed into a region of characteristic size $R$, a black hole forms if $R<R_{S}=2 G E$. If $R$ is identified with the "size" of an experiment and taken to be the De Broglie wavelength, which implies the scaling of size with energy $R \propto 1 / E$, then a black hole forms for any experiment at energies $E>E_{\text {Planck. }}$. Choptuik and Pretorius [2] have recently verified numerically that in classical general relativity the generic outcome of two particle scattering at small impact parameters indeed is a black hole. This vindicates the insights derived from the Aichelburg-Sexl result 3, 4] and other work on particle scattering [5[9].

Asymptotic darkness has interesting consequences for the structure of quantum field theory [10, 11], as well as for the status of gravity as an ordinary field theory in the UV. In particular, there is a relationship between black hole dominance and non-renormalizability of gravity. The scaling of black hole entropy with area implies that in asymptotically flat $d$-dimensions, the high energy density of states in black holes grows with energy as $e^{E^{\frac{d-2}{d-3}}}$. This scaling behavior then implies that quantum gravity cannot be related to a local conformal field theory in the UV, since in the latter case the universal scaling of density of states goes as $e^{E^{\frac{d-1}{d}}}$. The implication, as pointed out in ref. [12], is that gravity cannot be an ordinary renormalizable field theory, since a renormalizable field theory can be considered to be a perturbation of a conformal field theory by relevant operators, whose couplings at a given scale remain finite.

Asymptotic darkness also comes into play for some arguments proposing a minimum observable length [13]. If a black hole necessarily forms as one shrinks an experiment below the Planck length, then the Planck length serves as a minimum measurable distance as one cannot extract any information from the black hole. The existence of a fundamental length scale is considered by many a fairly model independent feature of quantum gravity 14 and could provide a fundamental cutoff to any quantum field theory.

In short, if asymptotic darkness is correct there are qualitatively new features for gravity in the UV that are not present if we simply think of gravity as a local quantum field theory of a spacetime metric. What then happens in a scenario for quantum gravity where gravity is supposed to be a well-behaved local quantum field theory in the UV as well as the IR? Such a scenario is envisioned by the asymptotic safety program, originally championed by 
Weinberg [15, 16]. Asymptotic safety can be described in the following heuristic way. Consider a truncated (for technical reasons, see [19]) effective action for 4-d metric gravity with cosmological constant coupled to matter

$$
\begin{array}{r}
I=\int d^{4} x \sqrt{-g}\left(-\Lambda(\mu)+\kappa_{0}(\mu) R+\kappa_{1}(\mu) R^{2}+\right. \\
\left.\kappa_{2}(\mu) R_{a b} R^{a b}+L_{m} \ldots\right)
\end{array}
$$

containing a finite set of higher derivative terms with associated coefficients, $\kappa_{1,2,3} \ldots$ in addition to the usual cosmological constant and EinsteinHilbert terms. $\mu$ is an energy scale associated with an experiment and the couplings are allowed to run with $\mu$. Since gravity couples to the matter stress-energy tensor, the actual strength of the gravitational coupling in a given experiment is (heuristically) totally controlled by $\mu$. It is therefore useful to consider the dimensionless coupling $\bar{\kappa}_{I}$ between gravity and matter generated by each term, $\bar{\kappa}_{I}=\mu^{d} / \kappa_{I}$, where $d=\left[\kappa_{I}\right]$, i.e the mass dimension of the coefficient (as opposed to the operator itself). So, for example, the Einstein-Hilbert term generates a dimensionless coupling $\bar{\kappa}_{0}=\mu^{2} / \kappa_{0}$, which is the familiar $G \mu^{2}$ interaction term. Higher curvature terms generate dimensionless couplings with lower powers of $\mu$. Some of these dimensionless couplings could blow up under the renormalization group flow of the $\kappa_{I}$ 's (with respect to the scale $\mu$ ). In particular, if $\kappa_{0}$ did not run, $\bar{\kappa}_{0}$ diverges as $\mu \rightarrow \infty$. In asymptotic safety, the dimensionless couplings remain finite and flow to a UV fixed point instead as $\mu \rightarrow \infty$. If this is true then metric gravity remains a valid, and in principle predictive description, down to arbitrarily short distances and high energies. While we do not know whether quantum general relativity is asymptotically safe, quite a few truncations offer evidence of a UV fixed point [17-21].

Since asymptotic safety requires that quantum gravity is a local field theory valid to arbitrarily high energies, while asymptotic darkness indicates that quantum gravity is not, there is an apparent tension between these two ideas. In this work we investigate this problem by reexamining the hoop conjecture in the context of the minimum length argument using one of the results of asymptotic safety - the running of coefficients such that $\bar{\kappa}_{I}$ remains finite. In particular we concern ourselves with the necessity of one or more horizons forming for an arbitrarily localized experiment and, more importantly, where they form. We then leverage a subtlety to the minimum length argument that allows us to perhaps reconcile asymptotic safety with asymptotic darkness for large black holes, as we now discuss.

Obviously, since any theory of quantum gravity must reduce to general relativity, a black hole must form for an experiment localized to scales much smaller than the Planck length as the necessary energy would be much greater than the Planck energy. The resulting black hole would be large compared to the Planck length and so governed by ordinary general relativity. This UV/IR connection has been pointed out by Banks among others [7, 11]. Formation of such a hole would certainly preclude transmission of any information generated by a localized experiment out to infinity, however it does not a priori imply that an experiment cannot locally measure arbitrarily small scales. Forbidding the measurement of arbitrarily small scales requires the horizon to be inside the region of the experiment. Consider for the moment pure general relativity and spherical symmetry, such that the resulting black hole is Schwarzschild. In this case any 2-sphere inside the black hole necessarily contains an apparent horizon from which information cannot escape and there is a minimum length. However, for Planck sized regions we are not in pure general relativity anymore - the gravitational constants may run significantly and higher curvature terms become relevant. Hence the small scale geometry may change significantly. If, for example, a pair of horizons formed outside a localized experiment then there would be no trapped surface inside the region of the experiment and therefore no minimum length. A highly localized experiment would still create a black hole as seen by an observer at infinity, but that is irrelevant to arguing that the experiment can not resolve distances below the Planck length.

For our simple thought experiment, consisting of a ball of uncharged matter inside a sphere $\Omega$ of proper radius $L$ with total energy $E \propto$ $1 / L$, the necessity of a horizon forming inside $\Omega$ as $L \rightarrow 0$ vanishes for asymptotic safety. There is no theorem that it doesn't form, and in fact whether it does or not depends on the size of higher curvature terms which are currently unmeasured and not uniquely calculated in asymptotic safety, but the strong argument from general relativity and quantum mechanics no longer holds. Hence the minimum length argument for localized experiments falls apart. We further conjecture that for $L<<L_{\text {Planck }}$ an interior horizon forms outside of $\Omega$ but with radius less than $L_{\text {Planck. }}$ While this would not necessarily have an effect on local experiments, it does mean that one cannot transmit information from the local experiment to infin- 
ity, as we noted above. The $L<<L_{\text {Planck }}$ states also have a large outer horizon that approaches the ordinary Schwarzschild horizon of general relativity. This is similar to the modified Schwarzschild solution of Reuter and Bonanno [22] that possesses an interior and exterior horizon. 35] In this way, the extreme UV behavior remains possibly compatible with both asymptotic safety and the results of general relativity for large black holes where quantum gravity is not important. A local experiment may not measure a minimum length, but we can't get that result back to our low energy, IR world that lives outside the outer horizon.

\section{THE HOOP "THEOREM" FOR SPHERICAL SYMMETRY}

Black hole dominance in the UV is a rather vague statement - there are myriad ways one could try and form black holes. However, if the hoop conjecture is correct, pretty much any way we concentrate enough matter into a small enough region should form one. Therefore we choose a specific behavior for gravity, our spacetime topology, and the distribution of matter as follows:

1. Gravity is asymptotically safe.

2. The spacetime topology is such that we can foliate spacetime into spacelike slices $\Sigma_{t}$, where $t$ is a time function.

3. A spherical ball $\Omega$ of proper radius $L$ is filled with matter with density $\rho$ on a maximal spacelike slice $\Sigma_{0}$.

4. The system is at least momentarily stationary.

If an experiment of proper radius $L$ is to exhibit a minimum length, then there must be at least an apparent horizon inside $\Omega$ as we shrink the size of the experiment. Our question is then whether an apparent horizon must be present on $\Sigma_{0}$ inside $\Omega$ in the limit as $L \rightarrow 0$.

We choose this particular setup for a number of reasons. First, the hoop conjecture in its usual form rests on explicitly using the Schwarzschild radius of a black hole. This poses several different problems. First, the event horizon is a global object. Further, the very existence of a sharply defined event horizon is questionable in a theory of quantum gravity. This observation has prompted much work on reformulating black hole physics in terms local trapping and/or dynamical horizons [23, 24]. We therefore use a more local reformulation of the conjecture in terms of trapped surfaces [25, 26]/apparent horizons [27 29]. Spherical symmetry and the momentarily stationary assumption are both used for simplicity. We note, however, that the stationary assumption also intuitively matches the idea of scaling a static system down to try to measure ever smaller distances in some frame.

The proof of Bizon et. al. 25], uses the Hamiltonian constraint from classical general relativity and constitutes a proof of the minimum length argument under certain conditions. We simply adjust the proof to include higher curvature terms and the running of coefficients. One might argue that the classical Hamiltonian constraint shouldn't be applied for questions about quantum gravity. Generically, there could be quantum corrections or other modifications to the Hamiltonian constraint at the Planck scale that are not captured by this simple semiclassical approach. However, we take the view that since we are asking how asymptotic safety can modify the standard minimum length argument, which is based on just such a semiclassical picture, the right thing to attempt first is to consider a specific prediction of asymptotic safety on a specific, mathematically precise formulation of the argument. A more problematic concern is specific to asymptotic safety itself, which is defined perturbatively. We use the results of a fully quantum mechanical but perturbative framework (where there is a well-defined Smatrix) to gain insight into a non-perturbative question, and there is no guarantee that nonperturbative effects can or should be neglected. However, this is again exactly what is done in the usual minimum length argument, where one treats an effective field theory (general relativity) that is only consistent in the low-energy perturbative regime classically even in the extreme UV. Hence for now we proceed in the same spirit and turn to the proof itself.

Consider a foliation of spacetime with Cauchy hypersurfaces denoted by $\Sigma_{t}$. Let $n^{a}$ be the normal to the leaves of the foliation. Choose $t=0$ and let $T_{M}^{a b}$ denote the matter stress tensor and $\rho=T_{M}^{a b} n_{a} n_{b}$ the energy density of a ball of matter in $\Sigma_{0}$. We assume the matter is instantaneously at rest inside a volume $\Omega$ of proper radius $L$ bounded by a 2 -surface $\partial \Omega$. The total proper energy of the matter, $E$, is defined by the following integral over proper volume $\Omega$,

$$
E=\int_{\Omega} T_{M}^{a b} n_{a} n_{b}=\int_{\Omega} \rho d v .
$$

$\partial \Omega$ has a unit spacelike normal $r^{a}$. The spatial metric $q_{a b}$ on $\Sigma_{0}$ and the extrinsic curvature $K^{a b}$ constitute canonical data. Whatever the 
particular truncation used, any higher curvature terms in eq. (1) contribute to the Hamiltonian constraint equation. We package the contribution of those terms into a single quantity $H_{\kappa}$. Then the Hamiltonian constraint equation for the pair $\left(q_{a b}, K^{a b}\right)$ is given by

$$
{ }^{3} R+K^{2}-K_{a b} K^{a b}=\kappa_{0}^{-1}\left(H_{\kappa}+\frac{1}{2} \rho\right) .
$$

Note that the coefficients of the higher curvature terms as they appear in the action eq. (11) are implicitly absorbed in $H_{\kappa}$ in eq. (3). We do not consider the contribution of the cosmological constant term to the Hamiltonian constraint. The implication of setting $\Lambda=0$ is discussed below when we review the renormalization group running of Newton's constant.

We first simplify using our assumptions. For a maximal slice that has everything momentarily stationary, the extrinsic curvature $K_{a b}$ (and any other single time derivative) vanishes. For spherical symmetry, a gauge can be chosen such that the metric $q_{a b}$ is conformally flat, $q_{a b}=\varphi^{4}(r) \delta_{a b}$. This in turn implies that the 3$\mathrm{d}$ Ricci scalar is given by ${ }^{3} R=-8 \varphi^{-5} \nabla^{2} \varphi$. For outgoing null rays emanating from any 2 -sphere inside $\Omega$ at radius $r$, the criterion that the 2 sphere is not a trapped surface is a condition on the null expansion

$$
\theta=r^{a} ; a>0 .
$$

Conversely, this must be less than or equal to zero if the 2-sphere is a trapped surface. With $r_{; a}^{a}=\left(r^{2} \varphi^{6}\right)^{-1} \frac{d\left(r^{2} \varphi^{4}\right)}{d r}$, the condition eq. (4) that the 2-sphere is not trapped is simply

$$
\theta_{r}=\partial_{r}\left(r \varphi^{2}\right)>0
$$

where the prime denotes an r-derivative.Finally, the proper radius of $\partial \Omega$ is $L=\int \varphi^{2} d r$.

Now, fix the 2-surface $\partial \Omega$ to have coordinate radius $r_{0}$ (and hence proper radius $\int_{0}^{r_{0}} \varphi^{2} d r$ ). Note that if the energy density due to matter and that of the higher curvature terms $\begin{array}{lll}30 & 32\end{array}$ is non-negative, then from the Hamiltonian constraint

$$
{ }^{3} R=-8 \varphi^{-5} \nabla^{2} \varphi=\kappa_{0}{ }^{-1}\left(H_{\kappa}+\frac{1}{2} \rho\right)
$$

we have that

$$
\nabla^{2} \varphi \leq 0
$$

which in turn implies that

$$
\frac{d \varphi}{d r} \leq 0
$$

As Bizon et al. argue, this condition is crucial in how the integrated constraints lead to eq. (13) (see below). This is rather obvious physically, as a negative energy density would certainly allow one to avoid creating a black hole. Integrating the Hamiltonian constraint over $\Omega$ we have,

$$
\begin{aligned}
& \int_{\Omega} d v\left(-8 \varphi^{-5} \nabla^{2} \varphi\right) \\
& =-\int_{0}^{r_{0}} 4 \pi \varphi^{6} r^{2} d r\left(8 \varphi^{-5} \nabla^{2} \varphi\right) \\
& =\left(2 \kappa_{0}\right)^{-1} \int_{\Omega} d v\left(\rho+2 H_{\kappa}\right) .
\end{aligned}
$$

The right hand integral is $1 / 2 \kappa_{0}$ times the total proper energy $E$ of the matter contained within $\Omega$ plus the contribution from the higher curvature terms. In spherical coordinates we then have

$$
-32 \pi \int_{0}^{r_{0}} d r \varphi \partial_{r}\left(r^{2} \partial_{r} \varphi\right)=\frac{1}{2 \kappa_{0}} E+\kappa_{0}^{-1} \int_{\Omega} d v H_{\kappa} .
$$

We can rewrite the left hand side of this equation as

$$
\begin{aligned}
& 16 \pi \int_{0}^{r_{0}} d r\left(\varphi^{2}-\partial_{r}\left(r \partial_{r}\left(r \varphi^{2}\right)\right)\right. \\
& \left.+r \varphi \partial_{r} \varphi+\varphi^{-1} r\left(\partial_{r} \varphi\right) \partial_{r}\left(r \varphi^{2}\right)\right)
\end{aligned}
$$

The first term of the integral is simply $16 \pi L$, where $L$ is the proper radius of $\Omega$. We hence have

$$
\begin{array}{r}
16 \pi L-\left.16 \pi r \partial_{r}\left(r \varphi^{2}\right)\right|_{d \Omega}(12) \\
+16 \pi \int d r\left(r \varphi \partial_{r} \varphi+\varphi^{-1} r\left(\partial_{r} \varphi\right) \partial_{r}\left(r \varphi^{2}\right)\right) \\
=\kappa_{0}^{-1}\left(\frac{E}{2}+\int_{\Omega} d v H_{\kappa}\right) .
\end{array}
$$

If there are no trapped surfaces in $\Omega$ and $\partial \Omega$ is not a trapped surface then by eqs. (5) and (8) every term except the first on the left hand side of (12) is negative. Therefore if there are no trapped surfaces in $\Omega$ we have the condition that

$$
16 \pi L>\kappa_{0}^{-1}\left(\frac{E}{2}+\int_{\Omega} d v H_{\kappa}\right) .
$$

The converse is also true - if this inequality is violated there is a trapped surface in $\Omega$. This then is the revised hoop condition that must be met by the mass and the integrated density of higher curvature terms.

In the case of pure general relativity, i.e. with $H_{\kappa}=0$ and no running of couplings, eq. (13) in conjunction with quantum mechanics implies 
that a trapped surface will inevitably form as the size of an experiment is shrunk. As we reduce the proper distance we are trying to resolve, $E$ must rise according to the uncertainty principle. If we trap an object in a region of proper distance $L, E$ scales as $L^{-1}$. In the limit as $L \rightarrow 0$ the LHS of eq. (13) goes to zero while $E$ diverges. Hence at some point the inequality cannot be satisfied and a trapped surface must form. This occurs at energy $E=32 \pi \kappa_{0} L \approx$ $32 \pi \kappa_{0} E^{-1}$, i.e. at energy $E \approx \sqrt{\kappa_{0}}=E_{\text {Planck }}$. At energies greater than $E_{\text {Planck }}$ the situation only gets worse, as a horizon is still present inside $\Omega$.

\section{IMPLICATIONS OF ASYMPTOTIC SAFETY}

In the asymptotic safety scenario of quantum gravity, Newton's constant, $G=\left(16 \pi \kappa_{0}\right)^{-1}$ is itself a running coupling (eq. 10). We first identify the energy scale $\mu$ of the running with $E$, the total proper energy in our ball of matter. This is of course not exactly true. Since we have a localized experiment there is no one particular energy we can assign to it (in contrast to a scattering scenario, for example). However, the total energy $E$ still is acceptable as a rough estimate of the energy scale we are probing spacetime at, especially since we are relating $E$ to the proper length $L$ via $E \sim 1 / L$, i.e. just interested in the scaling behavior.

A number of recent works in the literature [21] have confirmed the existence of a non-gaussian fixed point with various higher curvature truncations and matter contribution included in the total effective action. Integrating the flow equation is an involved process, but the upshot that we will exploit in the present article is that in the extreme UV, the running of Newton's constant is dominated by the fixed point behavior. This is given by

$$
\frac{1}{16 \pi \kappa_{0}(E)}=G(E) \simeq \frac{g_{*}}{E^{2}}
$$

where $g_{*}$ is the value of the dimensionless Newton's constant at the non-Gaussian fixed point, and we work in the regime where the matter proper energy exceeds the fiducial Planck energy set by the infrared value of Newton's constant. Thus in the extreme UV as $E^{2}$ increases, Newton's constant as a running coupling gets progressively smaller. We also note in our discussion here and what follows, the energy scale $E$ is to be compared with fiducial Planck scale $E_{p l}$ (used below) which is defined with respect to the infrared value of Newton's constant $G_{0}=\frac{1}{8 \pi E_{p l}^{2}}$.

\section{A. The cosmological constant}

Before we examine the effect of this behavior on the formation of local trapped surfaces, we would like to justify setting $\Lambda=0$. As discussed in existing literature [19], when we consider pure gravity, with say just the EinsteinHilbert term, Newton's constant is an inessential coupling and it can be changed by an overall field redefinition. Such redefinitions will leave the action invariant. Thus in the pure gravity sector, the running of Newton's constant is usually understood with reference to the running of some other fiducial operator in the action. The most commonly adopted choice for this reference operator is the cosmological constant term. In detail, the RG flow equation for the dimensionless Newton's constant

$$
E \frac{\partial g}{\partial E}=(2+\eta) g
$$

contains an anomalous dimension term $\eta(\Lambda)$. For the Einstein-Hilbert truncation this is given by

$$
\eta^{E H}=\frac{g B_{1}(\Lambda)}{1-g B_{2}(\Lambda)}
$$

Here $B_{1}(\Lambda)$ (respectively $B_{2}(\Lambda)$ ) are cosmological constant dependent functions which additionally also depend in a rather involved way on the details of how the infrared cut-off for the Exact Renomalization Group Equations (ERGE) is chosen. The anomalous dimension can be evaluated at zero arguments for these functions, $B_{1}(0)$ and $B_{2}(0)$, effectively restricting to the case of zero cosmological constant. In the case of the Einstein-Hilbert truncation the so-called optimized cut-off due to Litim [20] for instance leads to

$$
\eta^{E H}=\frac{12 g}{2 g-2\left(\Lambda-\frac{1}{2}\right)^{2}},
$$

hence setting $\Lambda=0$ in eq. (16) amounts to having

$$
\beta_{g}=\frac{(1-16 g) 2 g}{1-4 g}
$$

The behavior of Newton's constant gleaned from eq. (18) near the non-Gaussian fixed point $g_{*}=\frac{1}{16}$, for large $E$, is $G \simeq \frac{g_{*}}{E^{2}}$. Let us also note in passing that when higher curvature truncations are included in the effective action, the effect of these terms show up again in the anomalous dimension, now written as $\eta\left(\Lambda, H_{\kappa}\right)$. The fixed point behavior that this leads to has been discussed by Benedetti et al. [21]. 


\section{B. Running Newton's constant and the formation of trapped surfaces}

We now include the running of $\kappa_{0}$, eq. (14) into eq. (13). Momentarily restricting to the Einstein-Hilbert + matter truncation only we find the condition for no trapped surfaces inside $\Omega$ to be

$$
L>\frac{g_{*}}{2 E^{2}} E
$$

Now using the uncertainty relation, we replace $L \sim 1 / E$. The factors involving the proper energy $E$ drop out from both sides of the inequality eq. (19) and the (necessary) condition for no trapped surfaces to lie in $\Omega$ takes the form

$$
g_{*}<2
$$

This condition shows that up to $O(1)$ numbers arising from the order of magnitude estimates/scaling arguments we use in our derivation, there is some value of coefficients for which the minimum length argument falls apart, in contrast to ordinary general relativity. Interestingly, $g_{*}$ in different truncations has been found to be quite close to 2 . For example, the most recent results of Benedetti et al. [21], who consider both a minimally coupled scalar field with the Einstein-Hilbert action and a higher curvature truncation involving the Ricci and Weyl scalars, are

$$
\begin{array}{ll}
\left.g_{*}=0.860 \quad \text { (For Einstein-Hilbert truncation }\right) \\
g_{*}=2.279 \quad\left(\text { For } R^{2}+C^{2} \text { truncation }\right) \quad(21)
\end{array}
$$

In general the inclusion of higher curvature invariants in the effective action, constructed out of Ricci, Riemann and Weyl tensors, pushes the high energy fixed point towards values higher than their Einstein-Hilbert counterparts.

Consider now the impact of higher curvature terms with running couplings on our argument. Returning to eq. (13) we find that no trapped surfaces form inside $\Omega$ if

$$
L>\frac{g_{*}}{E^{2}}\left(\frac{E}{2}+\int d v H_{\kappa}\right)
$$

With $L$ replaced by $E^{-1}$ eq. (22) becomes

$$
E^{-1}>\frac{g_{*}}{E^{2}}\left(\frac{E}{2}+\int H_{\kappa} d v\right)
$$

We now observe that the integral involving the higher curvature terms is dimensionally an energy and so the integral must be a combination of $E$ multiplied by the dimensionless coefficients $\bar{\kappa}_{I}$. We therefore have,

$$
E^{-1}>g_{*}\left((2 E)^{-1}+E^{-1} \bar{I}\left(\bar{\kappa}_{I}\right)\right)
$$

where $\bar{I}\left(\bar{\kappa}_{I}\right)$ is a dimensionless number whose details depend on both the unknown $\kappa_{I}$ and the precise solution for the metric in the presence of the higher curvature terms with our given stress tensor. It suffices for us that it is a linear function of $\bar{\kappa}_{I}$. In asymptotic safety the $\bar{\kappa}_{I}$ coefficients remain finite as $E \rightarrow \infty$, and hence $I$ is also finite. Therefore as $E \rightarrow \infty$ there is no proof that a horizon is formed, as each side scales the same way with $E$, and so the argument for a minimum length no longer necessarily holds (at least in this formulation).

Of course, in the above approach, there is no proof that a horizon is not formed either. Since $E$ drops out in asymptotic safety at high energies, whether or not a horizon forms inside $\Omega$ depends on both the particulars of the value of $g_{*}$ at the non-Gaussian fixed point (and therefore on the truncation) and the actual size of the $\bar{\kappa}_{I}$ coefficients. Since both eq. (19) and eq. (24) are obtained in the extreme UV limit in which we invoke the largely scheme independent [33] fixed point dominated running eq. (14), our conclusions (the bound on the fixed point $g_{*}<2$, modulo $O(1)$ numbers discussed above) are at least essentially insensitive to myriad IRcutoff schemes in vogue and the precise size of the higher curvature terms at some fiducial infrared scale.

\section{GENERAL RELATIVITY AND THE INFRARED LIMIT}

While the question of whether or not a horizon forms inside $\Omega$ for Planck sized experiments becomes muddled with asymptotic safety, in the limit of energy much greater than $E_{P l}$ there still must be a trapped surface at large radius, as this corresponds to a large black hole with low horizon curvature (hence governed by general relativity). To reach such a spacetime picture in the semiclassical limit, one considers quantum states with low enough energy density that gravitational backreaction can be neglected propagating in a spacetime generated by some separate matteer distribution. For our superPlanckian experiments, we have no such luxury as we specifically are exploring a change in the gravitational action, the running of coefficients, via the energy scale of the experiment. However, we can analyze the "horizon" structure generated by a matter distribution within our framework by considering a background distribution of matter with total energy $E_{B}$ in $\Omega$ and ask when an experiment at a length scale $L_{E}$ (or equivalent energy scale $E_{E}$ ) encounters a trapped surface. 
If we wish to run our experiment all the way back to the infrared, we can no longer consider just the UV fixed point behavior for $G$. We therefore will take a specific form for $G(E) 22$, 34],

$$
G(E)=\frac{g_{*}}{E^{2}+g_{*} M_{P l}^{2}}
$$

and return to equation (13), but modify the scenario. We define

$F\left(E_{E}\right)=L_{E}-G\left(E_{E}\right)\left(\frac{E_{E}+E_{B}}{2}+\int d v H_{\kappa}\right)$

$F(E)>0$ is the condition for no trapped surface in the region. As $E_{E} \rightarrow \infty$ it dominates this expression, so we return to our original formulation of the hoop conjecture for a single experiment. We assume that $g_{*}$ and the $\kappa_{I}$ are such that there is no trapped surface within $\Omega$ as $E_{E} \rightarrow \infty$. Given this, as we $E_{E}$ towards the IR eventually $F\left(E_{E}\right)$ may cross zero. This signifies that at some radius $L_{E}$ we have crossed into a region that contains trapped surfaces. The inner boundary of this region can be thought of as an "inner horizon", similar to the ReissnerNordstrom inner horizon, although we caution that it is defined only as a function of the experiment energy $E_{E}$ and so is not a true spacetime quantity. Similarly, at very small $E_{E}, F\left(E_{E}\right)$ can cross zero again, signaling the outer boundary of our trapped surface region. This outer boundary surface corresponds to the black hole event horizon in the infrared.

We now solve for the location of these two boundary surfaces. The presence of a horizon is when

$$
L_{E}-G\left(E_{E}\right)\left(\frac{E_{E}+E_{B}}{2}+\int d v H_{\kappa}\right)=0
$$

$E_{E}$ and $L_{E}$ are related via $E_{E}=1 / L_{E}$, but $E_{B}$ is to be considered fixed. The contribution of the higher curvature terms can be reexpressed as $I_{E}\left(\kappa_{I}\right) E_{E}$ and $I_{B}\left(\kappa_{I}\right) E_{B}$, where each $I$ is again a dimensionless number dependent on the $\kappa_{I}$ 's. If we define $J_{E}=1 / 2+I_{E}$ and $J_{B}=1 / 2+I_{B}$ we can rewrite (27) in terms of the $J$ 's and $L_{E}$ as

$$
L_{E}-\frac{g_{*} L_{E}^{2}}{1+g_{*} L_{E}^{2} M_{P l}^{2}}\left(\frac{J_{E}}{L_{E}}+J_{B} E_{B}\right)=0 .
$$

Solving for $L_{E}$ yields
$L_{E}=\frac{g_{*} E_{B} J_{B} \pm \sqrt{g_{*}^{2} E_{B}^{2} J_{B}^{2}-4 g_{*} M_{P l}^{2}\left(1-g_{*} J_{E}\right)}}{2 g_{*} M_{P l}^{2}}$.

We now consider the "large black hole limit" in the infrared by assuming $E_{B}=N M_{P l}$ with $N>>1$, i.e. there's a lot of mass in our background spacetime. In this limit we have

$$
\begin{array}{r}
L_{E+}=\frac{E_{B} J_{B}}{M_{P l}^{2}} \\
L_{E-}=\frac{1-g_{*} J_{E}}{g_{*} E_{B} J_{B}} .
\end{array}
$$

$L_{E+}$ is $R \approx G E_{B}$, i.e. it corresponds to the Schwarzschild radius for a large black hole. $L_{E-}$ is the location of the inner horizon. We can get a more intuitive picture by setting all the higher curvature terms equal to zero. In this case $J_{E}=J_{B}=1 / 2$ and the inner horizon location becomes $L_{E-}=\left(2-g_{*}\right) /\left(g_{*} E_{B}\right)$. In the previous section, for just one experiment, our limit was roughly $g_{*}<2$ to avoid the minimum length argument. Here we have the additional background energy $E_{B}$ in addition to $E_{E}$, so we must further decrease $g_{*}$ to avoid a horizon forming in the domain of an experiment. In other words, if we chose $E_{E}=E_{B}$ for example, then there is twice the energy density as we had in the previous section in a region of size $L_{E}=L_{B}$ so $g_{*}$ would need to be less than one to prevent formation of a horizon. (Note that this experiment dependence of the limit on $g_{*}$ is symptomatic of this approach - whether and where horizons are formed depends strongly on how you plan to probe the spacetime as that influences the running of couplings.) If we consider $g_{*}<1$, such that this bound is satisfied, then we see that the inner horizon is reached when $L_{E} \approx 2 /\left(g_{*} E_{B}\right)$. We therefore have the following picture for $g_{*}<1$. We have a background energy density $E_{B}>>M_{P l}$ in a region of radius $L_{B}=1 / E_{B}$. We probe the system at length scales smaller than $L_{B}$ and we see no trapped surface. As we approach the length scale $\left(2-g_{*}\right) L_{B} / g_{*}$ we see a trapped surface form inside our experiment. This behavior persists until we reach the large radius $G E_{B}$, at which point we return to semiclassical physics outside an ordinary Schwarzschild horizon.

\section{CONCLUSIONS}

There's a rather obvious tension between black hole dominance and the asymptotic safety scenario of quantum gravity. The black hole 
dominance arguments indicate that the very high spectrum is dominated by states whose entropy density, via the Bekenstein-Hawking formula, does not scale as an extensive quantity with volume, which is a characteristic feature of any local conformal field theory. Since the high energy spectrum of renormalizable field theories are that of a conformal field theory, the implication is that gravity must be an exception to this rule. On the other hand, the asymptotic safety approach claims that precisely at those high energies where black holes dominate in the usual picture, a sensible local field theory based on the metric exists, and provides a reliable fundamental framework for quantum gravity.

Motivated by this, we re-examined the hoop conjecture in light of asymptotic safety. Adopting an approach relatively independent of any specific truncation of the effective action, we explored how in particular one key feature of asymptotic safety, the running of couplings, affects standard arguments for a minimum length based on the hoop conjecture when the size of an experiment is shrunk below a critical length. For a gedanken experiment consisting of a ball of matter inside a volume $\Omega$ of proper size $L$ and energy $E \sim \frac{1}{L}$, the fixed point behavior of the running couplings conspire such that the necessity of forming a trapped surface inside $\Omega$ as we shrink the size of an experiment vanishes. Hence the standard minimum length argument fails to necessarily be true in this case. However there is no proof in this approach that a trapped surface does not form inside $\Omega$ - whether it does or not depends on specifics of quantum gravity that we do not know.

The qualitative features of the fixed point dominated running and indeed the existence of a non-Gaussian fixed point has been argued by Percacci [33] to be a scheme independent feature of the the Wilsonian ERGE. He further notes that the position of the fixed point has a weak dependence on parameters in the choice of the IR cut-off function and so the values for the fixed points are thus believed to be quite robust. However, since our result depends on the fixed points in such a sensitive way, it is perhaps of general interest to extend the fixed point calculations to more realistic choices of matter Lagrangian and compare to a more detailed gedanken experiment.

Finally compatibility with general relativity can still be maintained even in our scenario. First, even if one can localize an experiment without formation of a trapped surface inside the region of the experiment, that is no guarantee that such microscopic information can be transmitted out to an observer at spatial infinity with physics dictated by general relativity. In fact, we found that no information about the local physics can be communicated to an observer at infinity, even if a local experiment inside that horizon can in principle measure arbitrarily small length scales. If we interpret asymtotic darkness as a statement about limitations on information transfer from a local experiment to an asymptotic observer owing to horizon formation, then our observations show that it may be possible to satisfy asymptotic darkness within the asymptotic safety scenario. Furthermore, for spacetimes with a background total energy $E \gg E_{p l}$, where the physics of general relativity dictate that there must exist a large horizon at the classical Schwarzschild radius, we argue that as one probes at lower and lower energy scales (from the UV to the IR) one eventually encounters a trapped surface. The resulting horizon structure consists of an experimentally dependent "inner horizon" and the classical Schwarzschild horizon in the extreme infrared.

\section{Acknowledgments}

We thank Jishnu Battacharyya and Keisuke Juge for comments and stimulating discussions.
[1] K. S. Thorne, in Magic Without Magic, ed. J. Klauder, (W. H. Freeman, San Francisco, 1972).

[2] M. Choptuik and F. Pretorius, 0908.1780 [grqc]

[3] P. D.D Éath and P. N. Payne, Phys. Rev. D46, 658 (1992); Phys. Rev. D46, 675 (1992); Phys. Rev. D 46, 694 (1992).

[4] P. C. Aichelburg and R. U. Sexl, Gen. Rel. Grav, 2, 303 (1971)

[5] S. B. Giddings and M. Srednicki, Phys. Rev. D
77, 085025 (2008)

[6] T. Banks and W. Fischler, eprint hep-th/9906038

[7] O. Aharony and T. Banks, JHEP, 9903:016 (1999).

[8] N. Kaloper and J. Terning, Gen. Rel. Grav, 39, 1525 (2007)[Int. J. Mod.Phys, D 17, 665 (2008)).

[9] D. M. Eardley and S. B. Giddings, Phys.Rev. D66, 044011 (2002).

[10] L. Crane and L. Smolin, Nucl. Phys. B267, 714 
(1986)

[11] Lecture available online hep.phys.uoc.gr/mideast2/lectures/BanksLecture.pdf

[12] A. Shomer, eprint Arxiv: 0709.3555.

[13] X. Calmet, M. Graesser and S. D. H. Hsu, Int. J. Mod. Phys. D14,2195,(2005).

[14] L. J. Garay, Int. J. Mod. Phys, A10, 145 (1995).

[15] S. Weinberg, in General Relativity: An Einstein Centenary Survey ed. S. W. Hawking and W. Israel, Cambridge University Press, Cambridge, 1979, pp. 790-831.

[16] S. Weinberg, arXiv:0908.1964 [hep-th].

[17] O. Lauscher and M. Reuter, JHEP 0510050 (2005), eprint hep-th/0508202.

[18] M. Niedermaier, Class. Quant. Grav. 24, R171 (2007), eprint gr-qc/0610018

[19] M. Niedermaier and M. Reuter, Living. Rev. Relativity, 9, 5 (2006)

[20] D. F. Litim, Phys. Rev. Lett. 92, 201301 (2004), eprint hep-th/0312114.

[21] D. Benedetti, P. Machado and F. Sauressig, Nucl.Phys.B824, 168 (2010), eprint ArXIv: 0902.4630 .

[22] A. Bonanno and M. Reuter, Phys.Rev.D62, 043008 (2000).

[23] A. Ashtekar, C. Beetle and S. Fairhurst, Class.Quant.Grav.16:L1-L7,1999. eprint gr-qc/9812065

[24] A. Ashtekar and B. Krishnan, Living
Rev.Rel.7:10,2004. eprint gr-qc/0407042

[25] P. Bizon, E.Malec and N O'Murchadha, Phys. Rev. Lett 61, 1147 (1988)

[26] E. Flanagan, Phys. Rev. D15, 1429 (1992)

[27] C. Barrabés, V. P. Frolov and E. Lesigne, Phys. Rev. D69, 19501(R) (2004)

[28] H. Yoshino and Y. Nambu, Phys. Rev. D67, 024009 (2003)

[29] T. Chiba, T. Nakamura, K. Nakao and M. Sasaki, Class. Quant. Grav. 11, 431 (1994).

[30] A. Strominger, Phys. Rev. D30, 2257 (1984)

[31] S. Deser and B. Tekin, Phys. Rev. D67, 084009 (2003)

[32] S. Deser and B. Tekin, Phys. Rev. D75, 084032(2007)

[33] G. Narain and R. Percacci, eprint:0910. 5390 [hep-th].

[34] JoAnne Hewett and Thomas Rizzo, JHEP 0712: 009 (2007)

[35] We caution the reader that in our approach the "inner horizon" is not a true spacetime inner horizon in the sense of a Reissner-Nordstrom inner horizon. It is instead rather experiment dependent, in that the location of the inner horizon depends on the total energy of the spacetime and the specific total energy of an experimental probe. 


\title{
Asymptotic Safety, Asymptotic Darkness, and the hoop conjecture in the extreme UV
}

\author{
Sayandeb Basu \\ Physics Department, University of the Pacific 3601 Pacific Avenue, Stockton, CA 9521目 \\ David Mattingly \\ Physics Department, University of New Hampshire, Durham, NH 0382
}

\begin{abstract}
Assuming the hoop conjecture in classical general relativity and quantum mechanics, any observer who attempts to perform a localized experiment in an arbitrarily small region will be stymied by the formation of a trapped surface within the spatial domain of the experiment. This thought experiment is frequently invoked in arguments for a fundamental minimum length in physics, which in turn is usually considered to be fairly independent of observer or experimental setup. We examine this conclusion in asymptotically safe gravity by modifying a proof of the hoop conjecture for spherically symmetric systems in general relativity to include higher curvature terms in the effective action as well as running couplings. We show that the modified proof fails, and so the argument for the mandatory formation of a trapped surface within the domain of an experiment also falls apart in this context. However, neither is there any contrary proof that local trapped surfaces do not form. Instead, in this approach whether or not an observer can perform local measurements in arbitrarily small regions depends on the specific numerical values of the couplings near the UV fixed point. In this sense, there is no longer any purely local version of the minimum length argument. However, when an experiment is localized to be much smaller than the Planck length we argue that at least one trapped surface must still form outside the experiment. This enshrouding horizon precludes any local information from reaching infinity and so there is still an effective minimum length for observers at infinity.
\end{abstract}

\section{INTRODUCTION}

The conjecture that the spectrum of particle states near the Planck scale is dominated by black holes, so called "asymptotic darkness", is an old one. Proof of such a conjecture requires a particular theory of quantum gravity, however one can make an argument solely with general relativity and quantum mechanics. The argument relies on Thorne's hoop conjecture [1]: If an amount of energy or matter $E$ is compressed into a region of characteristic size $L$, a trapped surface of size $L$ forms if $R<R_{S}=2 G E$. If $L$ is identified with the "size" of an experiment and taken to be the De Broglie wavelength, which implies the scaling of size with energy $R \propto 1 / E$, then a trapped surface forms for any experiment at energies $E>E_{\text {Planck. }}$. Choptuik and Pretorius [2] have recently verified numerically that in classical general relativity the generic outcome of two particle scattering at small impact parameters indeed is a black hole. This vindicates the insights derived from the Aichelburg-Sexl result [3, 44 and other work on particle scattering [5 [9].

Asymptotic darkness has interesting consequences for the structure of quantum field theory [10, 11], as well as for the status of gravity as an ordinary field theory in the UV. In particular, there is an obvious tension between black hole dominance and non-renormalizability of gravity. The scaling of black hole entropy with area implies that in asymptotically flat $d$-dimensions, the high

\footnotetext{
*Electronic address: sbasu@pacific.edu
}

${ }^{\dagger}$ Electronic address: dyo7@unh.edu energy density of states in black holes grows with energy as $e^{E^{\frac{d-2}{d-3}}}$. This scaling behavior then implies that quantum gravity cannot be related to a local conformal field theory in the UV, since in the latter case the universal scaling of density of states goes as $e^{E^{\frac{d-1}{d}}}$. The implication, as pointed out in ref. [12], is that gravity cannot be an ordinary renormalizable field theory, since a renormalizable field theory can be considered to be a perturbation of a conformal field theory by relevant operators, whose couplings at a given scale remain finite.

Our focus in this paper is on a different consequence of asymptotic darkness - its role in proposals for a minimum observable length [13] in physics. The logic is the following. If a trapped surface of size $L$ necessarily forms as one shrinks an experiment such that $L<L_{\text {Planck }}$, then the Planck length serves as a minimum measurable distance for any observer. To see why the conclusion is usually thought of as observer independent, consider simple general relativity and a static and spherically symmetric experiment. The solution at radii greater than $L$ is Schwarzschild, which has trapped surfaces at every radius from $L$ out to $2 G L^{-1}$. Therefore there is no observer at radius $L$ to infinity that could receive any signal from the experiment once it is scaled down past $L_{\text {Planck }}$ and hence $L_{\text {Planck }}$ becomes a fundamental measurement cutoff for any observer.

In short, if asymptotic darkness is correct there are qualitatively new features for gravity in the UV that are not present if we simply think of gravity as a local quantum field theory of a spacetime metric. What then happens in a scenario for quantum gravity where gravity is supposed to be a well-behaved local quantum field theory 
in the UV as well as the IR? Such a scenario is envisioned by the asymptotic safety program, originally championed by Weinberg [15, 16]. Asymptotic safety can be described in the following heuristic way. Consider a truncated (for technical reasons, see [19]) effective action for 4-d metric gravity with cosmological constant coupled to matter

$$
\begin{array}{r}
I=\int d^{4} x \sqrt{-g}\left(-\Lambda(\mu)+\kappa_{0}(\mu) R+\kappa_{1}(\mu) R^{2}+\right. \\
\left.\kappa_{2}(\mu) R_{a b} R^{a b}+L_{m} \ldots\right)
\end{array}
$$

containing a finite set of higher derivative terms with associated coefficients, $\kappa_{1,2,3 \ldots}$ in addition to the usual cosmological constant and Einstein-Hilbert terms. $\mu$ is an energy scale associated with an experiment and the couplings are allowed to run with $\mu$. Since gravity couples to the matter stress-energy tensor, the actual strength of the gravitational coupling in a given experiment is (heuristically) totally controlled by $\mu$. It is therefore useful to consider the dimensionless coupling $\bar{\kappa}_{I}$ between gravity and matter generated by each term, $\bar{\kappa}_{I}=\mu^{d} / \kappa_{I}$, where $d=\left[\kappa_{I}\right]$, i.e the mass dimension of the coefficient (as opposed to the operator itself). So, for example, the Einstein-Hilbert term generates a dimensionless coupling $\bar{\kappa}_{0}=\mu^{2} / \kappa_{0}$, which is the familiar $G \mu^{2}$ interaction term. Higher curvature terms generate dimensionless couplings with lower powers of $\mu$. Some of these dimensionless couplings could blow up under the renormalization group flow of the $\kappa_{I}$ 's (with respect to the scale $\mu$ ). In particular, if $\kappa_{0}$ did not run, $\bar{\kappa}_{0}$ diverges as $\mu \rightarrow \infty$. In asymptotic safety, the dimensionless couplings remain finite and flow to a UV fixed point instead as $\mu \rightarrow \infty$. If this is true then metric gravity remains a valid, and in principle predictive description, down to arbitrarily short distances and high energies. While we do not know whether quantum general relativity is asymptotically safe, quite a few truncations offer evidence of a UV fixed point [17 21].

Since asymptotic safety requires that quantum gravity is a local field theory valid to arbitrarily high energies, while asymptotic darkness indicates that quantum gravity is not, there is an apparent tension between these two ideas. In this work we investigate this problem by re-examining the hoop conjecture in the context of the minimum length argument using one of the results of asymptotic safety - the running of coefficients such that $\bar{\kappa}_{I}$ remains finite. In particular we concern ourselves with the necessity of one or more trapped surfaces forming for an arbitrarily localized experiment and, more importantly, where they form.

Obviously, since any theory of quantum gravity must reduce to general relativity in the infrared, a trapped surface must form somewhere for an experiment localized at $L<<L_{\text {Planck }}$ as the necessary energy would be much greater than the Planck energy. The resulting black hole would be large compared to the Planck length and so governed by ordinary general relativity. This UV/IR connection has been pointed out by Banks among others [7, 11]. Formation of such a large horizon would certainly preclude transmission of any information generated by a localized experiment out to infinity, however it does not a priori imply that an experiment cannot locally measure arbitrarily small scales. As mentioned above, forbidding the measurement of arbitrarily small scales for all observers requires a trapped surface form at the scale of the very localized experiment and this simply may fail in asymptotic safety. For Planck sized regions we are not in pure general relativity - the gravitational constants may run significantly and higher curvature terms become relevant. Hence the small scale geometry may change significantly and one may not have trapped surfaces all the way from a large outer horizon all the way down to the experiment size $L$ when $L<<L_{\text {Planck }}$.

For our simple thought experiment, consisting of a ball of uncharged matter inside a sphere $\Omega$ of proper radius $L$ with total energy $E \propto 1 / L$, the necessity of a trapped surface forming inside $\Omega$ as $L \rightarrow 0$ vanishes for asymptotic safety. There is no contrary statement that it doesn't form, and in fact whether it does or not depends on the size of higher curvature terms which are currently unmeasured and not uniquely calculated in asymptotic safety. However the strong argument from general relativity and quantum mechanics no longer holds. We further conjecture that for $L<<L_{\text {Planck }}$ a region of trapped surfaces forms outside of $\Omega$. This region has a large outer boundary that approaches the ordinary Schwarzschild horizon of general relativity. This is similar to the modified Schwarzschild solution of Reuter and Bonanno 22. that possesses an interior and exterior horizon. 37] In this way, the extreme UV behavior remains possibly compatible with both asymptotic safety and the results of general relativity for large black holes where quantum gravity is not important. A local experiment and local observers may not measure a minimum length, but that result cannot be transmitted back to any observers who live outside the outer horizon.

As a last introductory comment, we note that our construction is admittedly less than concrete in certain aspects and so should not be taken as a rigorous proof that asymptotically safe gravity generically avoids the consequences of asymptotic darkness. In particular, our results rely on a particular identification of the size of the experiment with the energy scale of the running couplings, and this is not concrete without setting up some specific experiment, similar to the scattering formalism used in Giddings, et. al. [36]. As well, shrinking an experiment to Planck size is also obviously impractical (and ignores the possibility of other new physics between here and the Planck scale). However, we chose this approach precisely to parallel the common and longstanding argument for a minimum length, which is taken to be very generic and model independent. Our goal is simply to show that this argument from general relativity no longer works locally and give a heuristic argument about how compatibility with general relativity in the infrared limit can still be maintained. A proof that asymptotic safety generically avoids the minimum length argument would require much more knowledge about the UV structure of asymptoti- 
cally safe gravity as well as constructing a specific, "best case" thought experiment, and so we leave that for future work.

\section{THE HOOP THEOREM FOR SPHERICAL SYMMETRY}

Black hole dominance in the UV is a rather vague statement - there are myriad ways one could try and form black holes. However, if the hoop conjecture is correct, pretty much any way we concentrate enough matter into a small enough region should form one. Therefore we choose a specific behavior for gravity, our spacetime topology, and the distribution of matter as follows:

1. Gravity is asymptotically safe.

2. The spacetime topology is such that we can foliate spacetime into spacelike slices $\Sigma_{t}$, where $t$ is a time function.

3. A spherical ball $\Omega$ of proper radius $L$ is filled with matter with density $\rho$ on a maximal spacelike slice $\Sigma_{0}$.

4. The system is at least momentarily stationary.

If an experiment of proper radius $L$ is to exhibit a minimum length, then there must be at least a trapped surface inside $\Omega$ as we shrink the size of the experiment. Our question is then whether a trapped surface must be present on $\Sigma_{0}$ inside $\Omega$ in the limit as $L \rightarrow 0$.

We choose this particular setup for a number of reasons. First, the hoop conjecture in its usual form rests on explicitly using the Schwarzschild radius of a black hole. This poses several different problems. First, the event horizon is a global object. Further, the very existence of a sharply defined event horizon is questionable in a theory of quantum gravity. This observation has prompted much work on reformulating black hole physics in terms local trapping and/or dynamical horizons [23, 24]. We therefore use a more local reformulation of the conjecture in terms of trapped surfaces [25, 26] /apparent horizons [27 29]. Spherical symmetry and the momentarily stationary assumption are both used for simplicity. We note, however, that the stationary assumption also intuitively matches the idea of scaling a static system down to try to measure ever smaller distances in some frame.

The proof of Bizon et. al. 25], uses the Hamiltonian constraint from classical general relativity and constitutes a proof of the minimum length argument under certain conditions. We simply adjust the proof to include higher curvature terms and the running of coefficients. One might argue that the classical Hamiltonian constraint shouldn't be applied for questions about quantum gravity. Generically, there could be quantum corrections or other modifications to the Hamiltonian constraint at the Planck scale that are not captured by this simple semiclassical approach. However, we take the view that since we are asking how asymptotic safety can modify the standard minimum length argument, which is based on just such a semiclassical picture, the right thing to attempt first is to consider a specific prediction of asymptotic safety on a specific, mathematically precise formulation of the argument. A more problematic concern is specific to asymptotic safety itself, which is defined perturbatively. We use the results of a fully quantum mechanical but perturbative framework (where there is a welldefined S-matrix) to gain insight into a non-perturbative question, and there is no guarantee that non-perturbative effects can or should be neglected. However, this is again exactly what is done in the usual minimum length argument, where one treats an effective field theory (general relativity) that is only consistent in the low-energy perturbative regime classically even in the extreme UV. Hence for now we proceed in the same spirit.

Consider a foliation of spacetime with Cauchy hypersurfaces denoted by $\Sigma_{t}$. Let $n^{a}$ be the normal to the leaves of the foliation. Choose $t=0$ and let $T_{M}^{a b}$ denote the matter stress tensor and $\rho=T_{M}^{a b} n_{a} n_{b}$ the energy density of a ball of matter in $\Sigma_{0}$. We assume the matter is instantaneously at rest inside a volume $\Omega$ of proper radius $L$ bounded by a 2 -surface $\partial \Omega$. The total proper energy of the matter, $E$, is defined by the following integral over proper volume $\Omega$,

$$
E=\int_{\Omega} T_{M}^{a b} n_{a} n_{b}=\int_{\Omega} \rho d v .
$$

$\partial \Omega$ has a unit spacelike normal $r^{a}$. The spatial metric $q_{a b}$ on $\Sigma_{0}$ and the extrinsic curvature $K^{a b}$ constitute canonical data. Whatever the particular truncation used, any higher curvature terms in eq. (1) contribute to the Hamiltonian constraint equation. We package the contribution of those terms into a single quantity $H_{\kappa}$. Then the Hamiltonian constraint equation for the pair $\left(q_{a b}, K^{a b}\right)$ is given by

$$
{ }^{3} R+K^{2}-K_{a b} K^{a b}=\kappa_{0}{ }^{-1}\left(H_{\kappa}+\frac{1}{2} \rho\right) .
$$

Note that the coefficients of the higher curvature terms as they appear in the action eq. (1) are implicitly absorbed in $H_{\kappa}$ in eq. (3). We do not consider the contribution of the cosmological constant term to the Hamiltonian constraint. The implication of setting $\Lambda=0$ is discussed below when we review the renormalization group running of Newton's constant.

We first simplify using our assumptions. For a maximal slice that has everything momentarily stationary, the extrinsic curvature $K_{a b}$ (and any other single time derivative) vanishes. For spherical symmetry, a gauge can be chosen such that the metric $q_{a b}$ is conformally flat, $q_{a b}=\varphi^{4}(r) \delta_{a b}$. This in turn implies that the 3-d Ricci scalar is given by ${ }^{3} R=-8 \varphi^{-5} \nabla^{2} \varphi$. For outgoing null rays emanating from any 2 -sphere inside $\Omega$ at radius $L$, the criterion that the 2 -sphere is not a trapped surface 
is a condition on the null expansion

$$
\theta=r^{a} ; a>0 \text {. }
$$

Conversely, this must be less than or equal to zero if the 2sphere is a trapped surface. With $r_{; a}^{a}=\left(r^{2} \varphi^{6}\right)^{-1} \frac{d\left(r^{2} \varphi^{4}\right)}{d r}$, the condition eq. (4) that the 2 -sphere is not trapped is simply

$$
\theta_{r}=\partial_{r}\left(r \varphi^{2}\right)>0
$$

where the prime denotes an r-derivative.Finally, the proper radius of $\partial \Omega$ is $L=\int \varphi^{2} d r$.

Now, fix the 2 -surface $\partial \Omega$ to have coordinate radius $r_{0}$ (and hence proper radius $\int_{0}^{r_{0}} \varphi^{2} d r$ ). Note that if the energy density due to matter and that of the higher curvature terms [30 32] is non-negative, then from the Hamiltonian constraint

$$
{ }^{3} R=-8 \varphi^{-5} \nabla^{2} \varphi=\kappa_{0}{ }^{-1}\left(H_{\kappa}+\frac{1}{2} \rho\right)
$$

we have that

$$
\nabla^{2} \varphi \leq 0
$$

which in turn implies that

$$
\frac{d \varphi}{d r} \leq 0
$$

As Bizon et al. argue, this condition is crucial in how the integrated constraints lead to eq. (13) (see below). This is rather obvious physically, as a negative energy density would certainly allow one to avoid creating a black hole. Integrating the Hamiltonian constraint over $\Omega$ we have,

$$
\begin{aligned}
& \int_{\Omega} d v\left(-8 \varphi^{-5} \nabla^{2} \varphi\right) \\
& =-\int_{0}^{r_{0}} 4 \pi \varphi^{6} r^{2} d r\left(8 \varphi^{-5} \nabla^{2} \varphi\right) \\
& =\left(2 \kappa_{0}\right)^{-1} \int_{\Omega} d v\left(\rho+2 H_{\kappa}\right) .
\end{aligned}
$$

The right hand integral is $1 / 2 \kappa_{0}$ times the total proper energy $E$ of the matter contained within $\Omega$ plus the contribution from the higher curvature terms. In spherical coordinates we then have

$$
-32 \pi \int_{0}^{r_{0}} d r \varphi \partial_{r}\left(r^{2} \partial_{r} \varphi\right)=\frac{1}{2 \kappa_{0}} E+\kappa_{0}^{-1} \int_{\Omega} d v H_{\kappa} .
$$

We can rewrite the left hand side of this equation as

$$
\begin{aligned}
& 16 \pi \int_{0}^{r_{0}} d r\left(\varphi^{2}-\partial_{r}\left(r \partial_{r}\left(r \varphi^{2}\right)\right)\right. \\
& \left.+r \varphi \partial_{r} \varphi+\varphi^{-1} r\left(\partial_{r} \varphi\right) \partial_{r}\left(r \varphi^{2}\right)\right)
\end{aligned}
$$

The first term of the integral is simply $16 \pi L$, where $L$ is the proper radius of $\Omega$. We hence have

$$
\begin{array}{r}
16 \pi L-\left.16 \pi r \partial_{r}\left(r \varphi^{2}\right)\right|_{d \Omega} \\
+16 \pi \int d r\left(r \varphi \partial_{r} \varphi+\varphi^{-1} r\left(\partial_{r} \varphi\right) \partial_{r}\left(r \varphi^{2}\right)\right) \\
=\kappa_{0}^{-1}\left(\frac{E}{2}+\int_{\Omega} d v H_{\kappa}\right) .
\end{array}
$$

If there are no trapped surfaces in $\Omega$ and $\partial \Omega$ is not a trapped surface then by eqs. (5) and (8) every term except the first on the left hand side of (12) is negative. Therefore if there are no trapped surfaces in $\Omega$ we have the condition that

$$
16 \pi L>\kappa_{0}^{-1}\left(\frac{E}{2}+\int_{\Omega} d v H_{\kappa}\right) .
$$

The converse is also true - if this inequality is violated there is a trapped surface in $\Omega$. This then is the revised hoop condition that must be met by the mass and the integrated density of higher curvature terms.

In the case of pure general relativity, i.e. with $H_{\kappa}=0$ and no running of couplings, eq. (13) in conjunction with quantum mechanics implies that a trapped surface will inevitably form as the size of an experiment is shrunk. As we reduce the proper distance we are trying to resolve, $E$ must rise according to the uncertainty principle. If we trap an object in a region of proper distance $L, E$ scales as $L^{-1}$. In the limit as $L \rightarrow 0$ the LHS of eq. (13) goes to zero while $E$ diverges. Hence at some point the inequality cannot be satisfied and a trapped surface must form. This occurs at energy $E=32 \pi \kappa_{0} L \approx 32 \pi \kappa_{0} E^{-1}$, i.e. at energy $E \approx \sqrt{\kappa_{0}}=E_{\text {Planck. }}$. At energies greater than $E_{\text {Planck }}$ the situation only gets worse, as a trapped surface is still present inside $\Omega$.

\section{IMPLICATIONS OF ASYMPTOTIC SAFETY}

In the asymptotic safety scenario of quantum gravity, Newton's constant, $G=\left(16 \pi \kappa_{0}\right)^{-1}$ is itself a running coupling (eq. 1). We first identify the energy scale $\mu$ of the running with $E$, the total proper energy in our ball of matter. This is of course not exactly true. Since we have a localized experiment there is no one particular energy we can assign to it (in contrast to a scattering scenario, for example). However, the total energy $E$ still is acceptable as a rough estimate of the energy scale we are probing spacetime at, especially since we are relating $E$ to the proper length $L$ via $E \sim 1 / L$, i.e. just interested in the scaling behavior.

A number of recent works in the literature [21] have confirmed the existence of a non-gaussian fixed point with various higher curvature truncations and matter contribution included in the total effective action. Integrating the flow equation is an involved process, but the upshot that we will exploit in the present article is that in the extreme UV, the running of Newton's constant is dominated by the fixed point behavior. This is given by

$$
\frac{1}{16 \pi \kappa_{0}(E)}=G(E) \simeq \frac{g_{*}}{E^{2}}
$$

where $g_{*}$ is the value of the dimensionless Newton's constant at the non-Gaussian fixed point, and we work in 
the regime where the matter proper energy exceeds the fiducial Planck energy set by the infrared value of Newton's constant. Thus in the extreme UV as $E^{2}$ increases, Newton's constant as a running coupling gets progressively smaller. We also note in our discussion here and what follows, the energy scale $E$ is to be compared with fiducial Planck scale $E_{p l}$ (used below) which is defined with respect to the infrared value of Newton's constant $G_{0}=\frac{1}{8 \pi E_{p l}^{2}}$.

\section{A. The cosmological constant}

Before we examine the effect of this behavior on the formation of local trapped surfaces, we would like to justify setting $\Lambda=0$. As discussed in existing literature [19], when we consider pure gravity, with say just the Einstein-Hilbert term, Newton's constant is an inessential coupling and it can be changed by an overall field redefinition. Such redefinitions will leave the action invariant. Thus in the pure gravity sector, the running of Newton's constant is usually understood with reference to the running of some other fiducial operator in the action. The most commonly adopted choice for this reference operator is the cosmological constant term. In detail, the RG flow equation for the dimensionless Newton's constant

$$
E \frac{\partial g}{\partial E}=(2+\eta) g
$$

contains an anomalous dimension term $\eta(\Lambda)$. For the Einstein-Hilbert truncation this is given by

$$
\eta^{E H}=\frac{g B_{1}(\Lambda)}{1-g B_{2}(\Lambda)}
$$

Here $B_{1}(\Lambda)$ (respectively $B_{2}(\Lambda)$ ) are cosmological constant dependent functions which additionally also depend in a rather involved way on the details of how the infrared cut-off for the Exact Renomalization Group Equations (ERGE) is chosen. The anomalous dimension can be evaluated at zero arguments for these functions, $B_{1}(0)$ and $B_{2}(0)$, effectively restricting to the case of zero cosmological constant. In the case of the Einstein-Hilbert truncation the so-called optimized cut-off due to Litim [20] for instance leads to

$$
\eta^{E H}=\frac{12 g}{2 g-2\left(\Lambda-\frac{1}{2}\right)^{2}},
$$

hence setting $\Lambda=0$ in eq. (16) amounts to having

$$
\beta_{g}=\frac{(1-16 g) 2 g}{1-4 g}
$$

The behavior of Newton's constant gleaned from eq. (18) near the non-Gaussian fixed point $g_{*}=\frac{1}{16}$, for large $E$, is $G \simeq \frac{g_{*}}{E^{2}}$. Let us also note in passing that when higher curvature truncations are included in the effective action, the effect of these terms show up again in the anomalous dimension, now written as $\eta\left(\Lambda, H_{\kappa}\right)$. The fixed point behavior that this leads to has been discussed by Benedetti et al. 21].

\section{B. Running Newton's constant and the formation of trapped surfaces}

We now include the running of $\kappa_{0}$, eq. (14) into eq.(13). Momentarily restricting to the Einstein-Hilbert + matter truncation only we find the condition for no trapped surfaces inside $\Omega$ to be

$$
L>\frac{g_{*}}{2 E^{2}} E
$$

Now using the uncertainty relation, we replace $L \sim 1 / E$. The factors involving the proper energy $E$ drop out from both sides of the inequality eq. (19) and the (necessary) condition for no trapped surfaces to lie in $\Omega$ takes the form

$$
g_{*}<2
$$

This condition shows that up to $O(1)$ numbers arising from the order of magnitude estimates/scaling arguments we use in our derivation, there is some value of coefficients for which the minimum length argument falls apart, in contrast to ordinary general relativity. Interestingly, $g_{*}$ in different truncations has been found to be quite close to 2. For example, the most recent results of Benedetti et al. 21], who consider both a minimally coupled scalar field with the Einstein-Hilbert action and a higher curvature truncation involving the Ricci and Weyl scalars, are

$$
\begin{array}{ll}
g_{*}=0.860 & (\text { For Einstein-Hilbert truncation }) \\
g_{*}=2.279 & \left(\text { For } R^{2}+C^{2} \text { truncation }\right)
\end{array}
$$

In general the inclusion of higher curvature invariants in the effective action, constructed out of Ricci, Riemann and Weyl tensors, pushes the high energy fixed point towards values higher than their Einstein-Hilbert counterparts.

Consider now the impact of higher curvature terms with running couplings on our argument. Returning to eq. (13) we find that no trapped surfaces form inside $\Omega$ if

$$
L>\frac{g_{*}}{E^{2}}\left(\frac{E}{2}+\int d v H_{\kappa}\right)
$$

With $L$ replaced by $E^{-1}$ eq. (22) becomes

$$
E^{-1}>\frac{g_{*}}{E^{2}}\left(\frac{E}{2}+\int H_{\kappa} d v\right)
$$

We now observe that the integral involving the higher curvature terms is dimensionally an energy and so the 
integral must be a combination of $E$ multiplied by the dimensionless coefficients $\bar{\kappa}_{I}$. We therefore have,

$$
E^{-1}>g_{*}\left((2 E)^{-1}+E^{-1} \bar{I}\left(\bar{\kappa}_{I}\right)\right)
$$

where $\bar{I}\left(\bar{\kappa}_{I}\right)$ is a dimensionless number whose details depend on both the unknown $\kappa_{I}$ and the precise solution for the metric in the presence of the higher curvature terms with our given stress tensor. It suffices for us that it is a linear function of $\bar{\kappa}_{I}$. In asymptotic safety the $\bar{\kappa}_{I}$ coefficients remain finite as $E \rightarrow \infty$, and hence $I$ is also finite. Therefore as $E \rightarrow \infty$ there is no proof that a trapped surface is formed, as each side scales the same way with $E$, and so the argument for a minimum length no longer necessarily holds (at least in this formulation).

Of course, in the above approach, there is no proof that a trapped surface is not formed either. Since $E$ drops out in asymptotic safety at high energies, whether or not a trapped surface forms inside $\Omega$ depends on both the particulars of the value of $g_{*}$ at the non-Gaussian fixed point (and therefore on the truncation) and the actual size of the $\bar{\kappa}_{I}$ coefficients. Since both eq. (19) and eq. (24) are obtained in the extreme UV limit in which we invoke the largely scheme independent [33] fixed point dominated running eq. (14), our conclusions (the bound on the fixed point $g_{*}<2$, modulo $O(1)$ numbers discussed above) are at least essentially insensitive to myriad IRcutoff schemes in vogue and the precise size of the higher curvature terms at some fiducial infrared scale.

\section{GENERAL RELATIVITY AND THE INFRARED LIMIT}

While the question of whether or not a trapped surface forms inside $\Omega$ for Planck sized experiments becomes muddled with asymptotic safety, in the limit of energy much greater than $E_{P l}$ there still must be a trapped surface at large radius, as this corresponds to a large black hole with low horizon curvature (hence governed by general relativity). To reach such a spacetime picture in the semiclassical limit, one considers quantum states with low enough energy density that gravitational backreaction can be neglected propagating in a spacetime generated by some separate matter distribution. For our super-Planckian experiments, we have no such luxury as we specifically are exploring a change in the gravitational action, the running of coefficients, via the energy scale of the experiment. However, we can analyze the "horizon" structure generated by a matter distribution within our framework by considering a background distribution of matter with total energy $E_{B}$ in $\Omega$ and ask when an experiment at a length scale $L_{E}$ (or equivalent energy scale $E_{E}$ ) encounters a trapped surface.

If we wish to run our experiment all the way back to the infrared, we can no longer consider just the UV fixed point behavior for $G$. We therefore will take a specific form for $G(E)$ [22, 34],

$$
G(E)=\frac{g_{*}}{E^{2}+g_{*} M_{P l}^{2}}
$$

and return to equation (13), but modify the scenario. We define

$$
F\left(E_{E}\right)=L_{E}-G\left(E_{E}\right)\left(\frac{E_{E}+E_{B}}{2}+\int d v H_{\kappa}\right)
$$

$F(E)>0$ is the condition for no trapped surface in the region. As $E_{E} \rightarrow \infty$ it dominates this expression, so we return to our original formulation of the hoop conjecture for a single experiment. We assume that $g_{*}$ and the $\kappa_{I}$ are such that there is no trapped surface within $\Omega$ as $E_{E} \rightarrow \infty$. Given this, as we $E_{E}$ towards the IR eventually $F\left(E_{E}\right)$ may cross zero. This signifies that at some radius $L_{E}$ we have crossed into a region that contains trapped surfaces. The inner boundary of this region can be thought of as an "inner horizon", similar to the Reissner-Nordstrom inner horizon, although we caution that in this framework it is defined only as a function of the experiment energy $E_{E}$ and so is not a true spacetime quantity. Similarly, at very small $E_{E}, F\left(E_{E}\right)$ can cross zero again, signaling the outer boundary of our trapped surface region. This outer boundary surface corresponds to the usual black hole event horizon in the infrared.

We now solve for the location of these two boundary surfaces. The presence of a horizon is when

$$
L_{E}-G\left(E_{E}\right)\left(\frac{E_{E}+E_{B}}{2}+\int d v H_{\kappa}\right)=0
$$

$E_{E}$ and $L_{E}$ are related via $E_{E}=1 / L_{E}$, but $E_{B}$ is to be considered fixed. The contribution of the higher curvature terms can be re-expressed as $I_{E}\left(\kappa_{I}\right) E_{E}$ and $I_{B}\left(\kappa_{I}\right) E_{B}$, where each $I$ is again a dimensionless number dependent on the $\kappa_{I}$ 's. If we define $J_{E}=1 / 2+I_{E}$ and $J_{B}=1 / 2+I_{B}$ we can rewrite (27) in terms of the $J$ 's and $L_{E}$ as

$$
L_{E}-\frac{g_{*} L_{E}^{2}}{1+g_{*} L_{E}^{2} M_{P l}^{2}}\left(\frac{J_{E}}{L_{E}}+J_{B} E_{B}\right)=0 .
$$

Solving for $L_{E}$ yields

$$
L_{E}=\frac{g_{*} E_{B} J_{B} \pm \sqrt{g_{*}^{2} E_{B}^{2} J_{B}^{2}-4 g_{*} M_{P l}^{2}\left(1-g_{*} J_{E}\right)}}{2 g_{*} M_{P l}^{2}} .
$$

We now consider the "large black hole limit" in the infrared by assuming $E_{B}=N M_{P l}$ with $N>>1$, i.e. there's a lot of mass in our background spacetime. In this limit we have

$$
\begin{array}{r}
L_{E+}=\frac{E_{B} J_{B}}{M_{P l}^{2}} \\
L_{E-}=\frac{1-g_{*} J_{E}}{g_{*} E_{B} J_{B}} .
\end{array}
$$


$L_{E+}$ is $R \approx G E_{B}$, i.e. it corresponds to the Schwarzschild radius for a large black hole. $L_{E-}$ is the location of the inner horizon. We can get a more intuitive picture by setting all the higher curvature terms equal to zero. In this case $J_{E}=J_{B}=1 / 2$ and the inner horizon location becomes $L_{E-}=\left(2-g_{*}\right) /\left(g_{*} E_{B}\right)$. In the previous section, for just one experiment, our limit was roughly $g_{*}<2$ to avoid the minimum length argument. Here we have the additional background energy $E_{B}$ in addition to $E_{E}$, so we must further decrease $g_{*}$ to avoid a trapped surface forming in the domain of an experiment. In other words, if we chose $E_{E}=E_{B}$ for example, then there is twice the energy density as we had in the previous section in a region of size $L_{E}=L_{B}$ so $g_{*}$ would need to be less than one to prevent formation of a trapped surface. (Note that this experiment dependence of the limit on $g_{*}$ is symptomatic of this approach - whether and where trapped surfaces form depends strongly on how you plan to probe the spacetime as that influences the running of couplings.) If we consider $g_{*}<1$, such that this bound is satisfied, then we see that the inner horizon is reached when $L_{E} \approx 2 /\left(g_{*} E_{B}\right)$.

We therefore have the following picture for $g_{*}<1$. We have a background energy density $E_{B}>>M_{P l}$ in a region of radius $L_{B}=1 / E_{B}$. We probe the system at length scales smaller than $L_{B}$ and we see no trapped surface. As we approach the length scale $\left(2-g_{*}\right) L_{B} / g_{*}$ we see a trapped surface form. This behavior persists until we reach the large radius $G E_{B}$, at which point we return to semiclassical physics outside an ordinary Schwarzschild horizon. Although current truncations have $g_{*}$ of $\mathrm{O}(1)$, it is interesting to note that if $g_{*}<<1$, the radius of the inner horizon can be much larger than the radius of the experiment, i.e. there is a relatively large region outside the experiment where no trapped surfaces exist. In this picture local observers performing local experiments see no trapped surfaces, and there is no minimum length for them. However, they cannot transmit any measurements all the way to spatial infinity. Hence for observers at asymptotic infinity (as would be the case in a scattering experiment for example) there is still a fundamental minimum observable length.

\section{CONCLUSIONS}

There's a rather obvious tension between the standard minimum length argument and asymptotic safety. Asymptotic safety predicts that gravity is renormalizable and hence well behaved into the extreme UV, whereas the minimum length argument predicts a fundamental cutoff to anny quantum field theory. Motivated by this, we re-examined the hoop conjecture in light of asymptotic safety. Adopting an approach relatively independent of any specific truncation of the effective action, we explored how in particular one key feature of asymptotic safety, the running of couplings, affects standard arguments for a minimum length based on the hoop conjecture when the size of an experiment is shrunk below a critical length. For a gedanken experiment consisting of a ball of matter inside a volume $\Omega$ of proper size $L$ and energy $E \sim \frac{1}{L}$, the fixed point behavior of the running couplings conspire such that the necessity of forming a trapped surface at the experimental scale vanishes. There is no proof in this approach that a trapped surface does not form inside $\Omega$ - whether it does or not depends on specifics of quantum gravity that we do not know. However the standard minimum length argument, that the Planck length is a minimum length scale, independent of observer or experiment, fails to necessarily be true in this case.

Compatibility with general relativity can still be maintained in this scenario. First, even if one can localize an experiment without formation of a trapped surface inside the region of the experiment, that is no guarantee that such microscopic information can be transmitted out to an observer at spatial infinity where the physics is dictated by general relativity. In fact, we found precisely this result: no information about the local physics can be communicated to an observer at infinity due to the formation of an enshrouding trapped surface. The resulting horizon structure consists of an energy dependent "inner horizon" and the classical Schwarzschild horizon in the extreme infrared. This difference between local measurements and asymptotic information necessitates caution when making blanket statements about the presence of a minimum length within the asymptotic safety approach 35]. One can, for example, instead address this question in a complementary way for scattering experiments 36] where the in/out states are at spatial infinity.

Finally, the qualitative features of the fixed point dominated running and indeed the existence of a non-Gaussian fixed point has been argued by Percacci 33. to be a largely scheme independent feature of the the Wilsonian ERGE. He further notes that the position of the fixed point has a weak dependence on parameters in the choice of the IR cut-off function and so the values for the fixed points are thus believed to be quite robust. However, since our result depends on the fixed points in such a sensitive way, it is perhaps of general interest to extend the fixed point calculations to more realistic choices of matter Lagrangian and compare to a more detailed gedanken experiment.

\section{Acknowledgments}

We thank Jishnu Battacharyya and Keisuke Juge for comments and stimulating discussions.
[1] K. S. Thorne, in Magic Without Magic, ed. J. Klauder, (W. H. Freeman, San Francisco, 1972).
[2] M. Choptuik and F. Pretorius, 0908.1780 [gr-qc] 
[3] P. D.D Éath and P. N. Payne, Phys. Rev. D46 , 658 (1992); Phys. Rev. D46, 675 (1992); Phys. Rev. D 46, 694 (1992).

[4] P. C. Aichelburg and R. U. Sexl, Gen. Rel. Grav, 2, 303 (1971)

[5] S. B. Giddings and M. Srednicki, Phys. Rev. D 77, 085025 (2008)

[6] T. Banks and W. Fischler, eprint hep-th/9906038.

[7] O. Aharony and T. Banks, JHEP, 9903:016 (1999).

[8] N. Kaloper and J. Terning, Gen. Rel. Grav, 39, 1525 (2007)[Int. J. Mod.Phys, D 17, 665 (2008)).

[9] D. M. Eardley and S. B. Giddings, Phys.Rev. D66, 044011 (2002).

[10] L. Crane and L. Smolin, Nucl. Phys. B267, 714 (1986)

[11] Lecture available online at hep.phys.uoc.gr/mideast2/lectures/BanksLecture.pdf

[12] A. Shomer, eprint Arxiv: 0709.3555.

[13] X. Calmet, M. Graesser and S. D. H. Hsu, Int. J. Mod. Phys. D14,2195,(2005).

[14] L. J. Garay, Int. J. Mod. Phys, A10, 145 (1995).

[15] S. Weinberg, in General Relativity: An Einstein Centenary Survey ed. S. W. Hawking and W. Israel, Cambridge University Press, Cambridge, 1979, pp. 790-831.

[16] S. Weinberg, arXiv:0908.1964 [hep-th].

[17] O. Lauscher and M. Reuter, JHEP 0510050 (2005), eprint hep-th/0508202

[18] M. Niedermaier, Class. Quant. Grav. 24, R171 (2007), eprint gr-qc/0610018

[19] M. Niedermaier and M. Reuter, Living. Rev. Relativity, $9,5(2006)$

[20] D. F. Litim, Phys. Rev. Lett. 92, 201301 (2004), eprint hep-th/0312114.

[21] D. Benedetti, P. Machado and F. Sauressig,
Nucl.Phys.B824, 168 (2010), eprint ArXIv: 0902.4630.

[22] A. Bonanno and M. Reuter, Phys.Rev.D62, 043008 (2000).

[23] A. Ashtekar, C. Beetle and S. Fairhurst, Class.Quant.Grav.16:L1-L7,1999. eprint gr-qc/9812065

[24] A. Ashtekar and B. Krishnan, Living Rev.Rel.7:10,2004. eprint gr-qc/0407042.

[25] P. Bizon, E.Malec and N O'Murchadha, Phys. Rev. Lett 61, 1147 (1988)

[26] E. Flanagan, Phys. Rev. D15, 1429 (1992)

[27] C. Barrabés, V. P. Frolov and E. Lesigne, Phys. Rev. D69, 19501(R) (2004)

[28] H. Yoshino and Y. Nambu, Phys. Rev. D67, 024009 (2003)

[29] T. Chiba, T. Nakamura, K. Nakao and M. Sasaki, Class. Quant. Grav. 11, 431 (1994).

[30] A. Strominger, Phys. Rev. D30, 2257 (1984)

[31] S. Deser and B. Tekin, Phys. Rev. D67, 084009 (2003)

[32] S. Deser and B. Tekin, Phys. Rev. D75, 084032(2007)

[33] G. Narain and R. Percacci, eprint:0910. 5390 [hep-th].

[34] JoAnne Hewett and Thomas Rizzo, JHEP 0712: 009 (2007)

[35] R. Percacci and G.-P. Vacca, arXiv:1008.3621 [hep-th]

[36] S. Giddings, M. Schmidt-Sommerfeld and J.R. Andersen 1005.5408 [hep-th].

[37] We caution the reader that in our approach the "inner horizon" is not a true spacetime inner horizon in the sense of a Reissner-Nordstrom inner horizon. It is instead rather experiment dependent, in that the location of the inner horizon depends on the total energy of the spacetime and the specific total energy of our experimental probe. 